\title{
La transición educativa del centro de desarrollo infantil al grado preescolar en Caucasia, Antioquia: ¿un proceso o un paso?*
}

\author{
The Educational Transition from the Child Development Center to the Preschool in Caucasia, \\ Antioquia: A Process or a Step? \\ Elizabeth Álvarez Fernández Yiceth Johana Rodríguez Bracamonte ${ }^{2}$
}

Para citar este artículo: Fernández, E., Rodríguez, Y. J. Recibido: 22-junio-2017 / Aprobado: 30-octubre-2017

(2018). La transición educativa del centro de desarrollo infantil al grado preescolar en Caucasia, Antioquia: ¿un proceso o un paso? Infancias Imágenes, 17(1), 67-77.

\section{Resumen}

Este artículo presenta un ejercicio de investigación cualitativa sobre el proceso de transición de los niños y niñas del centro de desarrollo infantil Los CameIlitos al grado preescolar de la institución educativa Santo Domingo del municipio de Caucasia (Antioquia, Colombia). Se realizó un análisis sobre las acciones docentes, administrativas y pedagógicas que favorecen o desfavorecen el proceso de transición en sus tres momentos (antes, durante y después) mediante entrevistas, observación no participante y análisis documental. Permitiendo así el reconocimiento de la articulación interinstitucional como elemento esencial para el proceso de transición, desde lo pedagógico y administrativo. Se tuvo en cuenta la formación de agentes educativos y docentes frente a la Guía ocho para transición como medio de orientación, del ICBF, para la transición de los niños y niñas a la educación formal. Este proceso se complementa con la recopilación de cuatro recomendaciones para un ejercicio de transición armoniosa.

Palabras clave: lineamiento; articulación; prácticas educativas; Instituto Colombiano de Bienestar Familiar (ICBF).

\begin{abstract}
This article presents a qualitative research exercise on the transition process of children from Los Camellitos child development center to the preschool level of Santo Domingo educational institution in the municipality of Caucasia (Antioquia, Colombia). An analysis was made of the teaching, administrative and pedagogical actions that favour or disfavour the transition process in its three moments (before, during and after) through interviews, non-participant observation and documentary analysis. Thus allowing the recognition of interinstitutional articulation as an essential element for the transition process, from the pedagogical and administrative viewpoint. The training of educational agents and teachers in front of Guía ocho para transición como medio de orientación, of the ICBF, for the transition of children to formal education was taken into account. This process is complemented by the compilation of four recommendations for a harmonious transition exercise.
\end{abstract}

Keywords: guidelines; articulation; educational practices; ICBF.

\footnotetext{
* La investigación realizada tiene por título: "La transición educativa de los niños y niñas del Centro de Desarrollo Infantil Los Camellitos del municipio de Caucasia al grado preescolar". Esta es de tipo cualitativo y está avalada por la Universidad de Antioquia; se llevó a cabo durante julio de 2016 y marzo 2017.

1 Psicóloga y licenciada en Pedagogía Infantil, Universidad de Antioquia. Correo electrónico: psicoudea2012@gmail.com

2 Licenciada en Pedagogía Infantil, Universidad de Antioquia. Correo electrónico: yiceth.rodriguez@udea.edu.co
} 


\section{Introducción}

Las transiciones son procesos de cambio que se experimentan en períodos críticos de la vida. Desde los momentos más remotos de la niñez, en el cambio que hace el cuerpo del mundo líquido, flexible y acogedor del vientre materno a la pesada y fría atmósfera de la tierra, hasta la vejez, con el paso de un cuerpo joven y vigoroso al de las limitaciones y los cuidados de los últimos años de vida; estas son etapas acompañas de miles de transiciones que se dan en diferentes ámbitos como el social, laboral, personal y, por supuesto, el escolar. Dichas transiciones representan un sinfín de experiencias, recuerdos y situaciones definitivas en los esfuerzos por integrarse a un nuevo grupo, en un nuevo ambiente, trascender en momentos críticos, experimentar cambios y, más aún, tener la capacidad de generarlos.

En el proceso que vivencian los niños y niñas que realizan su paso de la educación inicial a la formal (grado de transición) existen momentos y situaciones que pueden delimitarse como cruciales para el logro de una transición "armoniosa". En este caso, su recorrido inicia al salir del contexto familiar para llegar a la educación inicial, continúa en la educación formal (grado transición) y aspira a la prolongación de este proceso hasta la educación superior. Durante este periodo los niños y las niñas experimentan y se ven expuestos a cambios, los cuales significan crecimiento corporal, emocional, intelectual y cognitivo; en estos existe la posibilidad de experimentar sentimientos de pérdida, de inseguridad frente a lo desconocido. De igual forma, están acompañados de procesos de adaptación, que en algunos casos se dan de manera ágil, y en otros con un grado de dificultad. Lo anterior nos lleva a preguntar: ¿qué hace que para algunos niños y niñas el tránsito educativo sea un proceso armonioso y para otros no?

Por tal motivo, esta propuesta de investigación se centró en la transición educativa de los niños y niñas del centro de desarrollo infantil (CDI) Los Camellitos, del municipio de Caucasia, al grado preescolar (inicio de la educación formal), reconociéndola como un momento importante en los procesos de socialización de los niños y niñas que puede incidir a lo largo de todo su recorrido educacional. Se buscó comprender los procesos que acontecen durante la transición de los niños y niñas; identificar las características, situaciones o acciones asociadas a este proceso, que permiten describirlo como de fácil o difícil logro, entendiendo cómo la "particularidad" de las experiencias influye en el proceso de transición, sin dejar de lado el interés por explorar la participación de la escuela como espacio de educación (preescolar y básica primaria), siendo esta una zona con condiciones diferentes a la educación inicial.

\section{Metodología de investigación}

Este proyecto tiene un enfoque cualitativo, principalmente porque se identificó la naturaleza del fenómeno, su dinámica y sus manifestaciones. Cabe resaltar lo afirmado por Martínez (2006), "el enfoque cualitativo no tiene pretensiones de alta generalización de sus conclusiones, sino que, más bien, desea ofrecer resultados y sugerencias para instaurar cambios en una institución, en una empresa, en una escuela o en un grupo o comunidad particular" (p. 132).

A su vez, es de tipo fenomenológica pues en esta se buscaba describir el fenómeno de las transiciones, descubrir su esencia válida universalmente y útil científicamente. En palabras de Castaño y Quecedo: "busca comprender los fenómenos sociales desde la propia perspectiva del actor. Pretende comprender en un nivel personal los motivos y creencias que están detrás de las acciones" (2002, p. 7).

Por tal motivo, el diseño fenomenológico de esta propuesta investigativa se enfocó en las experiencias individuales subjetivas de los participantes (niños, niñas, docentes y directivos). Tratando de responder a la pregunta planteada por Salgado: “¿Cuál es el significado, estructura y esencia de una experiencia vivida por una persona (individual), grupo (grupal) o comunidad (colectiva) respecto de un fenómeno?" (2007, p.73); en este caso la transición educativa del CDI al grado transición.

Para la recolección de datos se utilizó la observación participante, la entrevista y el análisis 
documental de la guía orientadora $\mathrm{n}^{\circ} 8$ del ICBF (2014), además de las actas correspondientes a las reuniones de la mesa para transición del Bajo Cauca.

La población de estudio consta de dos centros educativos ubicados en el municipio de Caucasia: el CDI Los Camellitos y la institución educativa (IE) Santo Domingo.

El CDI Los Camellitos cuenta con 10 agentes educativas (mujeres), de las cuales tres se ocupan de los grupos que se encuentran en proceso de transición (compuestos por niños con edades que oscilan entre los 4 años con 5 meses y 4 años con 11 meses). Para el proceso de aplicación tanto de las entrevistas como de las guías de observación se tuvo en cuenta como variable principal que los grupos se encontraran en proceso de transición; para tal, la muestra fue de tres grupos que cumplieron con las condiciones de la variable. Para la clasificación y registro de las observaciones se denominarán grupo $\mathrm{O} 1, \mathrm{O} 2$ y $\mathrm{O} 3$; mientras que en el caso de las entrevistas estás serán E4, E5 y E6. En la investigación se entrevistó a más de 10 personas, cada una de ellas recibe una codificación; las presentadas acá son aquellas que realizan aportes al tema tratado para la presentación de dichos fragmentos de la investigación.

\section{Resultados}

Para la realización del análisis de los resultados se partió de las siguientes categorías de análisis: categoría \# 1 tránsito, emergiendo sub categoría concepciones de tránsito educativo. Sub categoría concepciones de tránsito armonioso; categoría \# 2 Prácticas: sub categoría - acciones educativas; categoría \# 3 lineamientos: sub categoría - articulación; categoría \# 4 recomendaciones. Definidas para sistematizar y organizar la información recolectada a través de la aplicación las entrevistas, las observaciones y el análisis documental (actas). En

\footnotetext{
3 El Instituto Colombiano de Bienestar Familiar (ICBF) es una entidad adscrita al Departamento Administrativo para la Prosperidad Social, antiguamente fue parte del Ministerio de la Protección Social. Fue creada en 1968 para dar respuesta a problemáticas como la deficiencia nutricional, la desintegración e inestabilidad de la familia, la pérdida de valores y la niñez abandonada.
}

este sentido, después de realizar una indagación con agentes educativos, docentes de preescolar, coordinadores académicos y psicólogos del CDI, se obtuvo lo que se evidencia en los siguientes apartados:

Categoría \# 1. Tránsito: concepciones de quien entrega y quien recibe

Se encuentra que la categoría de tránsito es definida como el paso de una etapa a otra en la que el niño o niña se prepara para ir al preescolar. En esta preparación se evidencian cambios a nivel físico y en los procesos de formación, relacionados no solo con el paso, sino con el cambio de modalidad, entorno, o grado, de la educación inicial a la educación formal. Esto es, por tanto, una preparación para la escuela que implica guiar, crecer, madurar y portar un conocimiento que les permita interactuar dentro de los nuevos grupos. Tal como lo evidencia la entrevista E2:

El paso de una etapa a otra, eso es transición, pasar de un estadio a otro estadio. (E2, 11/11/16)

Mientras que una profesora de preescolar, y coordinadora encargada en el año 2016, lo enuncia como:

El paso que tienen los niños que se encuentran en las modalidades o en la modalidad de atención de cero a siempre, hacia la educación formal, hacia el colegio [...]. (E7PC, 11/11/16)

Sin embargo, las entrevistadas realizan una diferenciación entre tránsito educativo y tránsito armonioso, entendiendo el primero como un paso de la educación inicial a la formal en el que se implica el crecimiento físico y emocional, determinado como madurez a través de la connotación de "niño grande" $^{\prime \prime}$; este nivel se define como un espacio con enfoque académico, es decir, en el que se hace énfasis en una formación más específica. De otra parte, el segundo tipo de tránsito se presenta en todas las etapas de la vida, desde el desarrollo evolutivo al cambio de contexto está acompañado de las acciones que garanticen los espacios y condiciones para lograr una adaptación. 
Así mismo, a partir de las observaciones realizadas se evidenciaron una serie de acciones preparatorias dentro de las acciones educativas docentes que acontecen en el proceso de transición del CDI Los Camellitos al preescolar de la IE Santo Domingo. En estas las docentes proponen actividades de representación del grado preescolar: "organizando el espacio semejando el aula de clases del preescolar, con sillas y mesas en grupos, pizarrón o tablero y cajas de colores y hojas blancas sobre cada escritorio" (O1).

Categoría \# 2. Prácticas educativas: actividades o procesos

En esta categoría se pudo encontrar aquellas prácticas que se relacionan directamente con las acciones educativas que se llevan a cabo tanto en el CDI como en la IE, antes, durante y después del proceso de tránsito. Es decir, son acciones que tienen un sentido, una intención, y que se convierten en aspectos que favorece o desfavorece el tránsito.

Dichas se clasifican en acciones educativas Ilevadas a cabo en los tres momentos del proceso de transición (antes, durante, y después).

Las acciones educativas son definidas como todas aquellas acciones que buscan ofrecer al niño y la niña experiencias de interacción que les permitan adquirir autonomía, independencia y actitudes fundamentales para desarrollar habilidades que permitan enfrentar el cambio de un escenario a otro. En este sentido, una de las entrevistadas afirma que las acciones que desarrolla buscan:

Brindarle al niño conocimientos de cómo interactuar ellos en grupo, cómo defenderse ellos en otro contexto que no es el de aquí, para que ellos sean autónomos y sean independientes, sean capaces de estar solos, no acompañados por uno. Que uno les brinde que es lo que ellos van a ver más allá, que es interactuar con niños más grandes, que por lo tanto debemos de tener en cuenta lo principal: tener una buena convivencia, un respeto no solo conmigo mismo sino con los demás. (E6, 24/11/16)

Por ello, las acciones descritas por las entrevistadas se clasifican en acciones antes, durante y después.
Acciones antes del proceso de transición

Son aquellas acciones propuestas por las agentes educativas y las docentes de preescolar antes del proceso de transición. Estas se enfocan en la realización de actividades pedagógicas de aprestamiento, como el uso del cuaderno, el manejo del reglón, el recortado y la pre-escritura; además de actividades lúdicas y tareas, reforzadas con un diálogo preparatorio que afianza el paso a la otra etapa, especialmente para evitar dificultades de desenvolvimiento del niño en el nuevo espacio, como lo ejemplifica una de las entrevistadas:

Las actividades que yo trabajo van enfocadas con ese fin, que en el colegio no les dé duro manejar un cuaderno, que nos les dé duro coger el color, porque ya se está trabajando con ellos que deben aprender a no salirse de las márgenes [...] para que no sea traumático para los niños cuando vayan al colegio. (E4, 24/11/16)

Dentro de las acciones educativas se evidencia cómo las agentes educativas y docentes de prescolar abordan la preparación para el tránsito a través de la representación, actividades de dramatizado y juegos de roles sobre cómo sería un día de clases en el grado transición. Estas buscan permitir al niño prepararse para los cambios que representa el paso al preescolar, incorporando elementos como son el cuidado de los objetos personales, los tiempos para el aprendizaje, el juego y la alimentación. Tal como lo manifiesta la entrevistada E6:

Bueno, por ejemplo, el juego, las lecturas de cuento, el dramatizado, que hacemos con niños referente a los cuentos, que ellos sean capaces de meterse en ese cuento que nosotros le hemos compartido y que sean capaces ellos de formar sus conocimientos dependiendo de lo que nosotros les brindemos. (E6, 24/11/16)

\section{Acciones durante el proceso de transición}

Como su nombre lo indica, son todas aquellas acciones que desarrollaron las agentes educativas y docentes de preescolar enmarcadas en la acción pedagógica principal realizada durante la preparación para el proceso de transición: 
[...] la pasantía (visita) de los niños y niñas del CDI al preescolar de la institución educativa Santo Domingo. (A1, 07/24/16)

Se destaca la importancia de que los niños de ambos grupos compartieran la experiencia, realizaran un reconocimiento de la institución y de algunas de las docentes de prescolar que los recibirían el siguiente año escolar, como refiere la docente entrevistada E4:

Tuvimos una visita a la institución Santo Domingo, [...] la profesora les mostró las instalaciones, subieron, la profe les leyó un cuento, estuvieron sentados en las sillas, se les dio a conocer cómo trabajaban, qué comparten en las mesas, [...], allá trabajan un poquito más y juegan un poquito menos. (E4, 24/11/16)

\section{Acciones después del proceso de transición}

Son todas aquellas acciones docentes y administrativas llevadas a cabo después del tránsito de los niños y niñas del CDI al grado preescolar, orientadas en el acompañamiento y enfocadas en la realización de actividades lúdico-pedagógicas para que los niños y niñas se sientan incluidos en la institución y en el seguimiento de su tránsito a través del Simat (Sistema Integrado de Matrícula):

Bueno de los niños que transitaron estamos obligados a hacer un seguimiento y es enviar todo el listado de los niños de todos los CDI de aquí de Caucasia, todos los que transitaron, enviarlo a Secretaría de Educación y verificar que todos los niños efectivamente estén matriculados, que sí estén registrados en el Simat [...]. (EPsico-2017)

Durante el ejercicio de las entrevistas las participantes nombran y ejemplifican acciones docentes que favorecen o desfavorecen el tránsito escolar, dentro de las cuales destacan como acciones favorecedoras todo aquello relacionado con el papel tanto de las agentes educativas del CDI como de las docentes de preescolar en el acompañamiento a los niños y niñas.

Por otra parte, en el grupo de las acciones desfavorecedoras las entrevistas destacan la falta de un recibimiento personalizado a los niños y niñas por parte de la docente o agente educativa y la falta de empatía hacia ellos:

Entonces, ¿qué puede pasar negativo en ellos? Como el recibimiento. ¿Sí me entiende? También como el allá va a hacer tareas, jallá va a hacer tareas!, no porque de todas maneras un preescolar es un preescolar, hay que hacer tareas, pero tiene que ser con mucha dedicación y cuidadito para no fastidiarlos, por decirlo así. (E4, 24/11/16)

Categoría \# 3. Lineamiento: articulación acciones de engranaje

En esta categoría se evidenció, tanto en los discursos como en la observación y análisis de las actas, las diferentes relaciones que se establecen en las articulaciones entre las instituciones (CDI-IE) y lo determinado por los lineamientos del ICBF (2014). Este último, en concreto, en el conocimiento que tienen agentes educativas y docentes del documento.

Esto hace necesario, por tanto, el reconocimiento de la articulación como una subcategoría que agrupa toda la acción que implica una interacción entre la institucionalidad y los miembros que la componen.

En el proceso de tránsito de los niños y niñas del CDI Los Camellitos al grado preescolar de la IE Santo Domingo se llevaron a cabo acciones enmarcadas en la guía, la cual:

Brinda lineamientos generales frente a la articulación que debe instarse entre los diferentes sectores para la transición de todos los niños y niñas atendidos por los programas de primera infancia del ICBF, al sector educativo. (E3, 11/11/16)

En estos, se circunscribe la categoría lineamiento, el cual alude a todos aquellos aspectos, acciones y procesos de articulación entre los profesionales (docentes, agentes educativos, directivos, psicólogos, etc.) y las instituciones en torno a la generación de:

[...] estrategias de articulación de los procesos pedagógicos para la transición de los niños y niñas de las modalidades de atención del ICBF al grado obligatorio del preescolar. (E3, 11/11/16) 
Guía que para muchas de las entrevistadas es desconocida, tal como lo manifiesta E1, E2, E3, E4, E6 y E7PC, y especialmente E4, en la siguiente afirmación:

[...] mmm no, nosotras no tenemos conocimiento de la guía, de los lineamientos del ICBF, no nos han dado una capacitación respecto a eso. Manejamos actividades desde la corporación, pero no manejamos directamente los lineamientos. (E4, 24/11/16)

La articulación se define como las acciones de engranaje y correlación de los procesos que tanto el recurso humano como las instituciones realizan en la formación del niño para el favorecimiento del tránsito al grado preescolar, tal como lo afirman las entrevistadas:

Es correlacionar, es decir, lo que enseñan. Un ejemplo: saber cómo viene el niño de la guardería, o los CDI, para nosotras luego seguir ese proceso en el grado preescolar. (E1, 11/11/16)

Es el engranaje de una etapa a la otra, de un periodo a la otra, de un estadio a otro. (E2, 11/11/16)

En esta investigación fue posible encontrar que para una efectiva articulación también se desarroIlan acciones antes, durante y después del proceso de tránsito que pueden favorecer o desfavorecer lo que acontece durante dicho proceso. Estas acciones se relacionan directamente con la orientación dada desde las políticas, en este caso lo establecido en la guía $n^{\circ} 8$ del ICBF (2014), comprendidas como las reuniones entre representantes de una de las EAS (Entidades Administradoras de Servicios) y representantes del ICBF. Las acciones durante se relacionan con la pasantía y evidencian desconocimiento de lo articulación pedagógica y el poco conocimiento del proceso de enseñanza-aprendizaje entre ambas instituciones; además de la falta de relación entre docentes y agentes educativas.

Acciones de articulación antes del proceso de transición

A partir de lo consignado en las actas y en la información obtenida en las entrevistas, en el proceso de tránsito educativo del CDI Los Camellitos al grado preescolar de la IE Santo Domingo, en las acciones de articulación Ilevadas a cabo antes del inicio del proceso de tránsito, se desarrollaron acciones enmarcadas en lo establecido en por el ICBF (2014), lo cual:

Brinda lineamientos generales frente a la articulación que debe instarse entre los diferentes sectores para la transición de todos los niños y niñas atendidos por los programas de primera infancia del ICBF, al sector educativo. (A3, 05/06/16)

Acciones de articulación durante el proceso de transición

La articulación durante en proceso de tránsito ha estado delegado a la realización de dos visitas guiadas, denominadas por las instituciones como pasantías.

La primera donde los niños y niñas del CDI (tres grupos) próximos a transitar participaron de actividades lideradas por las docentes de la institución educativa, y algunos [...] estudiantes [del grado preescolar]. (A1, 07/25/16)

Esto con el objetivo de la:

Exploración del nuevo contexto a través del recorrido de las dependencias del plantel educativo [...] coordinación, sala de informática, biblioteca, sala de maestros, comedor, tienda y cafetería. (A1, 07/25/16)

De igual forma, es posible identificar que no hay un conocimiento en relación a los procesos de enseñanza aprendizaje y a lo trabajado en cada escenario, como lo evidencia una agente educativa:

Bueno, llevamos los primeros pinitos, pero realmente nos faltaría; a nosotros nos faltaría conocer más el proceso que llevan allá, y a ellos también el proceso que tenemos aquí. (E3, 11/11/16)

Acciones de articulación después de la transición De las acciones de articulación posteriores al tránsito, las entrevistadas manifiestan que el proceso ha sido satisfactorio por la capacidad de adaptación de los niños y niñas al nuevo espacio y por la 
capacidad de ellos de responder a los compromisos de la escolaridad.

Sin embargo, respecto a las acciones articuladas que se han desarrollado en el año 2017 entre el CDI y la IE, entre docentes y agentes, las entrevistadas afirman que no ha habido ninguna clase de articulación o acercamiento.

Ninguna. No conozco a ninguna de las profesoras de allá, solo a la que estuvo el día de la visita. (E4, 24/11/16)

Dentro de este proceso de articulación hay unos factores que favorecen y desfavorecen las transiciones educativas de los niños y niñas. En los factores favorecedores pueden destacarse la actitud del recurso humano involucrado en la atención y formación de los niños y niñas frente al proceso, el acompañamiento de los padres y la articulación entre los docentes y las instituciones. Tal como lo afirma la docente E1:

Yo creo que sobre todo la familia. Los niños cuando empiezan pues esa etapa necesita mucho de los padres para ellos sentirse apoyados, amados. (E1, 11/11/16)

Mientras que dentro de los factores que desfavorecen, las participantes nombran y ejemplifican, aparte de las acciones docentes nombradas en el apartado anterior, "Acciones educativas docentes que favorecen o desfavorecen lo que acontecen antes, durante y después del proceso de tránsito", dentro de dichas acciones desfavorecedoras, vinculan a los agentes, padres de familia entre otro.

Categoría \# 4. Recomendaciones: retos del nuevo proceso

En esta categoría es posible encontrar los saberes de las participantes (supuestos o desde lo que conocen de la guía $n^{\circ} 8$ ), en relación a sus prácticas diarias y de la introyección de los lineamientos, una serie de propuestas que retoman la articulación de las condiciones que pueden permitir un proceso de transición armonioso.

Despues de la indagación con agentes educativas, docentes de preescolar, coordinadores académicos y psicólogos del CDI, se encuentra que esta categoría se conforma de aquellas acciones, aspectos y procesos que para las entrevistas favorecen el tránsito armonioso que se enmarcan en cuatro ejes principales:

\section{Proceso de sensibilzción y acompañamiento a} los padres de familia: para que estos cuenten con herramientas y aprendizajes que les permitan realizar un adecuado acompañamiento a los niños y niñas que inician su proceso de transicion, tal como lo afirma la siguiente entrevistada:

Yo diría que para completar el tránsito sería la vinculación de los padres de familia [...]. Yo creo que me adelanté un poquito, pero creo que tendría que ver con la participación de los papás. (E3, 11/11/16)

2. Articulación entre sectores, instituciones y actores: a traves de la cual se realize un acompañamiento enfocado en el antes, durante y después del proceso de transición, por medio del desarrollo de actividades que garanticen a los niños y niñas un tránsito armonioso. En este sentido afirman:

Hacer integración entre los niños que están acá con los niños que vienen, las docentes, de pronto los padres de familia también invitarlos a que conozcan las instituciones, a que conozcan el PEI que estamos trabajando. (E2, 11/11/16)

\section{Procesos de formación o cualificación conjun-} ta: dirigidos a agentes educativos y docentes de preescolar a propósito de la propuesta de una mesa de trabajo que permita un ejercicio de articulación a través del cual se genere una relación hilvanada entre los procesos desarrollados en el CDI y el preescolar.

Que las docentes del CDI y la psicóloga hicieran unas actividades previas con nosotros para que pudiéramos lograr hacer algo más efectivo, o lo que esperan de pronto que se haga. (E3, 11/11/16)

\section{Revisión y reconstrucción de los lineamien- tos para el proceso de tránsito: enfocado}


principlamente en el proceso de revisión de los lineamientos establecidos para el proceso de tránsito, la vinculación del Estado (representado por el ICBF) y la Secretaría de Educación y su responsabilidad como eje articulador del proceso de transición. Tal como lo manifiesta E2:

Primero que todo, el ICBF organice o se articule con las instituciones educativas oficiales para mirar las políticas de trabajo que ellas manejan en los CDI. Porque los CDI, usted sabe, son nuevos en el municipio, apenas están arrancando; entonces no hacer solo el proceso de trabajo con los docentes de allá, sino también con nosotros que somos las institución receptora, y no solo que sea el ICBF, sino también las instituciones educativas. Esto debe ser un propósito de la Secretaría de Educación Municipal, que haga ese tipo de articulaciones o que propicie los espacios, porque esa es la mayor dificultad: que se propicien los espacios para que nos encontremos los de CDI con las instituciones educativas. (E2, 11/11/16)

\section{Discusión}

El tránsito es comprendido como el cambio que hacen los niños y niñas de un lugar o fase de la educación a otro a través del tiempo. Este representa desafíos desde el punto de vista de las relaciones sociales, el estilo de enseñanza, el ambiente, el espacio, el tiempo, los contextos de aprendizaje y el aprendizaje mismo, haciendo del proceso algo intenso y con demandas crecientes. No se puede desconocer que "el cambio puede ser o una oportunidad para aprendizajes nuevos o puede ser la causa de aprensión frente a lo nuevo que genera confusión y ansiedad, sensaciones que pueden afectar el comportamiento de un individuo a largo plazo" tal como lo afirma Abello (2008, p. 22). Pues, aunque todos los niños de una forma u otra transitan, "en algunos casos, es con tensiones y a través de situaciones difíciles y con resultados mediocres, y en otros, la transición es interesante, aportadora y por tanto, exitosa" (Peralta, 2007, p. 8). En este sentido, cuando nos referimos a una "transición en educación", nos estamos refiriendo al proceso intermedio que transcurre entre un estado que es definido social y culturalmente como "inicial" y el cual se hay que superar por otro, que hay que alcanzar (p. 4).
Sin embargo, para comprender lo anterior es necesario identificar que el concepto de tránsito se encuentra sujeto a dos adjetivos. El primero, denominado tránsito educativo o transición educativa, el cual se entiende como el cambio o paso de la educación inicial a la formal en el que se implica el crecimiento físico y emocional, determinado como madurez, a través de la connotación "niño grande", que:

En concordancia con el planteamiento de Bronfenbrenner, [...] considera al niño como el actor principal de su propio desarrollo en la medida en que, a partir de sus condiciones y características personales, resignifica y transforma las experiencias que le ofrecen las distintas interacciones en los contextos en los cuales se desarrolla. (Carvalhol, 2015, p. 20)

El segundo adjetivo es el tránsito armonioso, el cual tiene la connotación de ser algo permanente en las diferentes etapas del desarrollo evolutivo e incluye los cambios en el contexto. Estos son comprendidos como parte del tránsito armonioso que están directamente relacionados con las acciones ejecutas $u$ omitidas por quienes se ocupan de garantizar los espacios y condiciones para lograr una adaptación. De esta manera, las agentes educativas se refieren al tránsito armonioso como el conjunto de etapas que se van dando en la vida en todos los aspectos. Dicho de otra forma, se trata del paso de un estadio a otro.

En la educación inicial, las acciones de las agentes educativas enfocadas en beneficio del proceso de tránsito son aquellas que le ofrecen al niño herramientas para enfrentar los desafíos y exigencias de la escuela, desarrolladas a partir de actividades lúdico-pedagógicas y enmarcadas en dos grandes enfoques:

- Las que ofrecen al niño o niña la adquisición de habilidades como el uso del cuaderno, del lápiz, el manejo del reglón, el recortado, el rasgado, la pre-escritura, entre otras.

- Las que permiten el desarrollo de cualidades sociales y emocionales como el saber compartir, cuidar sus pertenencias, ser responsables, entre otras. 
Actividades que en general ofrecen al niño y la niña experiencias de interacción en las que adquieren conocimientos, autonomía, independencia, actitudes fundamentales para desarrollar habilidades de convivencia y saberes necesarios para que el proceso de transición sea menos traumático. Esto a partir del diálogo preparatorio que afianza el paso a la otra etapa, especialmente para evitar dificultades de desenvolvimiento del niño en el nuevo espacio. Especialmente porque, tal como lo afirma Camargo:

Educar en la primera infancia significa proponer, por parte de los distintos miembros de la sociedad, acciones conducentes a lograr la inmersión de las nuevas generaciones en la cultura, que contribuyan a su estructuración como seres sociales que aprenden a convivir con otros. $(2014$, p. 44)

Pero, de otra parte, en la educación formal las acciones de las docentes se enfocan en el acompañamiento y la realización de actividades lúdico-pedagógicas para que los niños y niñas se sientan incluidos dentro de la IE. Sin embargo, en lo concerniente a la sensibilización de agentes educativos y docentes a partir de los lineamientos generales brindados por el ICBF (2014), puede decirse que han sido desligados del proceso de tránsito llevado a cabo, tal como lo evidencia el desconocimiento por parte de las entrevistadas de las orientaciones de la guía. Esto desfavorece de manera directa la labor que cumplen las docentes y las agentes en pro del tránsito de los niños y niñas.

Cabe resaltar que, aunque las entrevistadas manifiestan el desconocimiento de la guía del ICBF (2014), puede decirse que sus acciones no estuvieron muy alejadas de lo que esta plantea. Sin embargo, aún hay aspectos por interiorizar y reflexionar respecto al desarrollo del proceso que se pone en práctica, especialmente porque el mismo Ministerio de Educación Nacional (MEN, 2016), afirma que "el grado Transición no se ofrece de acuerdo a los lineamientos de la política de atención integral del Gobierno Nacional", lo que se ve reflejado en los índices de deserción y repitencia en los primeros grados obligatorios del sistema. "En Colombia 7.778 niños y niñas desertan en transición, es decir el $3,25 \%$ frente a $2,83 \%$ que lo hacen en primaria.
Quienes más repiten año escolar son aquellos que pasan de preescolar al grado primero con un 2,72\%" (MEN, 2016). Todo esto evidencia los vacíos y las repercusiones de un desconocimiento de las implicaciones del proceso de transición en la vida escolar.

Por otra parte, el seguimiento del proceso de tránsito desde la vía operativa y administrativa, se limita a la verificación de un cupo a través del Simat; y en caso:

[...] de que se presente pues un caso en el que haya un niño que no esté registrado, que no esté yendo al colegio, que ya tenga la edad para estar en el colegio, se hace la devolución, o se hace la solicitud también con Secretaría de Educación y con las entidades pertinentes para hacer un seguimiento, primero se hace visita domiciliaria para verificar que está pasando, por qué se fueron, qué fue lo que les pasó, por qué el niño no está matriculado, y empezamos un proceso de seguimiento sobre por qué se le estaría vulnerando un derecho del niño, el derecho a la educación. (EPsico-2017)

Así las cosas, tanto la guía del ICBF (2014), como las acciones y criterios del proceso de tránsito de los niños y niñas del CDI Los Camellitos al grado preescolar de la IE Santo Domingo Ilevado a cabo entre el año 2016-2017, demuestran que la base para la transición al sistema educativo es la vinculación del niño y la niña a partir de los cinco años de edad. Pues: "se evidencia que la mayor parte de los esfuerzos, acciones y recursos se concentra en garantizar la matrícula efectiva del total de los niños y las niñas que han cumplido la edad para el ingreso al grado de transición" (ICBF, 2014, p. 7).

\section{Conclusiones}

Dado que el municipio de Caucasia solo inicia hasta el año 2016 su proceso de reconocimiento e implementación de la guía del ICBF, como estrategia de orientación para los proceso de transición de los niños y niñas que pasan del CDI al grado preescolar, se puede pensar que dicha situación da lugar a la oportunidad de construir y adoptar una propuesta articulada y contextualizada a través de la cual se reconozcan las propuestas existentes a nivel nacional como "Todos listos ya" y "Transición es una nota". Esto a raíz de la ausencia de conocimiento 
por parte de las docentes y agentes educativas en relación a la guía establecida como marco referencial para el proceso de tránsito.

Para lo anterior se requiere un cambio o reorientación de los procesos de articulación interinstitucional en el que se pueda clarificar las formas de intervención y los procesos que generen un ejercicio de acción conjunta entre las instituciones y entidades encargadas de la educación inicial y la educación formal. Lo anterior debería permitir desde la institucionalidad la gestión de recursos, la construcción de una propuesta de transición a nivel municipal, el reconocimiento del docente y su labor, las necesidades de acompañamiento y formación a la familia como fuente primaria para el apoyo socio-emocional, y a los niños y niñas como sujetos activos en el proceso de transición. De la mano de una preparación (cualificación-experiencia) docente que conciba nuevas estrategias de formación y construcción de experiencia que le permitan a las agentes, docentes, psicólogos y cuerpo directivo de las instituciones contar con las herramientas necesarias para comprender el proceso de transición desde los diferentes niveles que lo conforman. Es decir, desde el desarrollo emocional, cognitivo y social en los niños; a nivel familiar como núcleo reproductor de los aprendizajes; y a nivel institucional con la articulación profesional.

En este postulado final queremos dejar por sentado que a pesar de lo desalentador que pueda parecer el panorama frente a los procesos de transición de los niños y niñas, existen elementos que pueden transformarse en el puente para la construcción de nuevos procesos a través de los cuales se pueda dar un nuevo sentido a la preparación para el tránsito y el trabajo articulado de las institucionalidades para el logro de la armonía en el mismo. Ejemplo de ello es el reconocimiento que hacen las participantes en su discurso de las faltas dentro de su proceso de formación y acción pedagógica para atender las implicaciones estratégicas, pedagógicas y emocionales que implica la transición. Otro elemento alentador es la construcción colectiva de las líneas de trabajo o recomendaciones que se transforman en posibilitadores de un tránsito armonioso. Estos son insumos para atrevernos a afirmar que apostar por la construcción de un nuevo marco de acciones pedagógicas institucionales es la alternativa para que el municipio de Caucasia construya su plan de acompañamiento a los procesos de transición de manera contextualizada y coherente con las necesidades de los niños y niñas, agentes, docentes, familiares e institucionalidad.

A través de los ejercicios observación y entrevista se amplía la perspectiva fundamentada por los agentes entrevistados sobre el sentimiento de desconocimiento frente a las directrices o propuestas para el proceso de transición, la poca claridad conceptual de tránsito educativo y tránsito armonioso, la desarticulación de los procesos pedagógicos que se desarrollan desde el CDI y el preescolar. Esto da como resultado un proceso de transición fragmentado en el que cada uno de los sujetos a cargo genera actividades encaminadas desde perspectivas diferentes que ocasionalmente tienen puntos de encuentro, pero que a los ojos de un lector externo no definen un sentido integrativo del proceso de transición y, con mayor deficiencia, aún no se evidencia en los niños y niñas un proceso real de preparación y adaptación al cambio. La falta de cualificación y formación sobre el proceso de transición deviene en la construcción y ejecución de un listado de chequeo de actividades que no logran trascender del sentido del hacer productos como loncheras, planas, reconocimiento de vocales y números; no se evalúa el impacto que generan las mismas sobre las necesidades de adaptación del niño, de su preparación para el cambio, del sentimiento de pérdida o angustia que puede generar el enfrentarse al discurso del adulto que informa de la terminación de un ciclo (que se reconoce como la pérdida de la tranquilidad, de los cuidados maternales, del sustento nutricional), a la ganancia del título de crecimiento o madurez y las responsabilidades que implica iniciarse en el aprendizaje académico.

Lo anterior nos lleva a pensar el lugar que ocupan los niños y niñas dentro de este proceso de transición, incluso desde la forma como son nombrados en el discurso de las participantes de esta investigación. Con la revisión global de los contenidos de las entrevistas y actas se puede afirmar que en un $99 \%$ los discursos apuntan a las necesidades de articulación, cualificación, participación de los grupos familiares, intervención institucional y la 
garantía del cupo escolar; pero menos del 1\% del discurso realiza un reconocimiento de las emociones que acompañan o expresan los niños y niñas en el proceso de tránsito. Ello se evidencia en expresiones como: "a ellos les gusta mucho y se sienten satisfechos con las actividades". El cuestionamiento a este argumento se basa en la imposibilidad de delimitar cuáles son los medios que utiliza la docente para determinar si estas expresiones del discurso dan cuenta en realidad de experiencia emocional que los niños y niñas vivencian durante la transición; cuando en la planeación y en las acciones pedagógicas no se registran espacios para la expresión emocional, para el reconocimiento frente a las emociones de desagrado o incluso de interés frente a los imaginarios que niños y niñas construye sobre lo que implica ir a la escuela. Evidencia de esto es que en los diferentes momentos del discurso de las participantes solo se define uno en el cual se reconocen las emociones negativas expresadas por algunos niños con respecto a la pérdida del acompañamiento de las docentes en su ingreso al preescolar. Lo cual se puede considerar como un aspecto egocéntrico, pues solo se logra reconocer la emoción desde la implicación de la docente, pero no se evidencia el lugar del niño como sujeto consciente, activo y participativo, pues es él quien experimenta la transición como autor de los referentes emocionales y cognitivos que lo acompañan en este proceso, para fundamentar sus estrategias de adaptación a futuros cambios.

Es necesario afirmar que no existen en el discurso de las participantes de esta investigación un lugar para el desarrollo vivo de estos niños y niñas; el lugar que ocupan, en otras palabras, es el de ser el objeto en formación que pasa de un ambiente a otro que requiere de unos cuidados y saberes que le garanticen el llegar a ser un sujeto pensante capaz de responder a las demandas educativas que sobre su ser recaen.

\section{Referencias}

Abello, R. (2008). Transiciones al inicio de la escolaridad en una institución educativa de carácter privado en Bogotá: una experiencia de construcción de sentido. Manizales, Colombia: Universidad de Manizales, Centro de Estudios Avanzados en Niñez y Juventud alianza de la Universidad de Manizales y el Cinde.

Camargo, M. (2014). Sentido de la educación inicial. Bogotá: MEN. Recuperado de https://www. educacionbogota.edu.co/archivos/Temas $\% 20$ estrategicos/Educacion inicial/2016/Sentido EducacionInicial.pdf

Carvalhol, G. (comp.) (2015). Modelo de transiciones exitosas en la primera infancia fundación Bancolombia. Bogotá: Fundación Bancolombia y Alina Consultorías. Recuperado de http:// www.alinaconsultorias.com/guia-transiciones-exitosas-primera-infancia.pdf

Castaño, C. y Quecedo, R. (2002). Introducción a la metodología de investigación cualitativa. Revista de Psicodidáctica, 14, 5-39.

Instituto Colombiano de Bienestar Familiar (ICBF) (2014). Gestión para la atención integral a la primera infancia. Guía orientadora.$^{\circ} 8$. Guía para la transición de los niños y niñas desde los programas de atención a la primera infancia del ICBF al sistema de educación formal. Recuperado de https://www.icbf.gov.co/sites/default/ files/procesos/g13. pp guia orientadora de transito de ninas y ninosdesde los programas de atencion a la primera infancia del icbf sistema educativo.pdf

Martínez, M. (2006). La investigación cualitativa (síntesis conceptual). Revista de Investigación en Psicología, 9(1), 123-146. https://doi. org/10.15381/rinvp.v9i1.4033

Ministerio de Educación Nacional (MEN) (2016). Guía metodológica para la elaboración del Plan Sectorial de Educación. Recuperado de https://www.mineducacion.gov.co/1759/articles-356185 foto portada.pdf

Peralta, M. V. (2007). Transiciones en educación Infantil: un marco para abordar el tema de calidad. Washington D.C.: Organización de Estados Americanos.

Salgado, A. C. (2007). Investigación cualitativa: diseños, evaluación del rigor metodológico y retos. Liberabit, 13(13), 71-78. 